\title{
ON A CLASS OF MAGNETIC SCHRÖDINGER OPERATORS WITH DISCRETE SPECTRUM
}

\author{
N. ANGHEL \\ (Communicated by Varghese Mathai)
}

\begin{abstract}
We introduce a class of magnetic Schrödinger operators in $\mathbf{R}^{n}$ which exhibit pure point spectrum in a fashion that is actually easy to check. This class is an adequate generalization of the more familiar two-dimensional setting, and the proof we give for its spectral discreteness is novel, based on the use of Euclidean Dirac operators coupled to vector potentials.
\end{abstract}

\section{INTRODUCTION}

Let $H_{a}=\sum_{j=1}^{n}\left(\frac{1}{i} \frac{\partial}{\partial x_{j}}+a_{j}\right)^{2}, i=\sqrt{-1}$, be a magnetic Schrödinger operator in $\mathbf{R}^{n}, n \geq 2$, with coordinates $x=\left(x_{1}, x_{2}, \ldots, x_{n}\right)$, where $a=\sum_{j=1}^{n} a_{j} d x_{j}$ is a magnetic potential whose components $a_{j}=a_{j}(x)$ are real-valued functions of class $C^{1}$. It is well known [AHS, I] [KS that $H_{a}$ with domain $C_{0}^{\infty}\left(\mathbf{R}^{n}, \mathbf{C}\right)$ is an essentially selfadjoint operator in $L^{2}\left(\mathbf{R}^{n}, \mathbf{C}\right)$. Its spectrum, which is non-negative, depends only on the magnetic field $B=d a$ associated to $a$,

$$
B=\sum_{j<k} B_{j k} d x_{j} \wedge d x_{k}, \quad B_{j k}=\frac{\partial a_{k}}{\partial x_{j}}-\frac{\partial a_{j}}{\partial x_{k}},
$$

by gauge invariance $\underline{\mathrm{AHS}}, \mathrm{I}, \mathrm{KS}$.

It is a matter of great interest to decide for which magnetic fields $B, H_{a}$ admits a discrete spectrum, that is, a spectrum consisting only in isolated eigenvalues of finite multiplicity. Although sufficient conditions for this to happen are known, mainly involving the behavior of $|B|=\left(\sum_{j<k} B_{j k}^{2}\right)^{1 / 2}$ at infinity under a controlled rate of change of the directions $B_{j k}$ of $B$ [AHS, D, HM, I, KS, no satisfactory necessary and sufficient conditions are available.

The purpose of this paper is to present yet another sufficient condition for the discreteness of the spectrum of $H_{a}$, which seems to be the natural generalization of the familiar case $n=2$. Its advantage is that it hides the need to control the rate of change of the directions of $B$ in a format easily verifiable in concrete situations. Also, its proof, based on the use of Euclidean Dirac operators coupled to vector potentials, differs from those presented so far in the literature.

Received by the editors January 5, 2011.

2010 Mathematics Subject Classification. Primary 35J10; Secondary 35P05, 47F05, 81V10.

Key words and phrases. Schrödinger operator, magnetic field, discrete spectrum, Dirac operator. 
Theorem. Let $H_{a}$ be a magnetic Schrödinger operator in $\mathbf{R}^{n}$ with magnetic field $B$, as described above. Assume that there are distinct indices $j_{1}<k_{1}, j_{2}<k_{2}, \ldots, j_{\mu}<$ $k_{\mu}, \mu=\left[\frac{n}{2}\right]$, in the interval $[1, n]$, and a sequence of signs $\pm 1, \epsilon_{1}, \epsilon_{2}, \ldots, \epsilon_{\mu}$, such that

$$
\lim _{|x| \longrightarrow \infty} \sum_{p=1}^{\mu} \epsilon_{p} B_{j_{p} k_{p}}(x)=+\infty .
$$

Then $H_{a}$ has a discrete spectrum in $L^{2}\left(\mathbf{R}^{n}, \mathbf{C}\right)$.

\section{Euclidean Dirac operators COUpled to VeCtor POTENTIALS}

On $\mathbf{R}^{n}, n \geq 2$, consider an irreducible representation of the complex Clifford algebra $C l\left(\mathbf{R}^{n}\right)$ on the finite-dimensional Hermitian complex vector space $\mathbf{C}^{2^{\mu}}$, $\mu=\left[\frac{n}{2}\right]$. This is equivalent to the prescription of $n$ skew-Hermitian endomorphisms of $\mathbb{C}^{2^{\mu}}, E_{1}, E_{2}, \ldots, E_{n}$ such that for every $j, E_{j}^{2}=-I d$, and $E_{j} E_{k}+E_{k} E_{j}=0$ for every $j \neq k$. For instance, such a concrete representation, useful for our needs, is the following:

Define first the $2 \times 2$ complex matrices $U, V$, and $W$, by

$$
U=\left[\begin{array}{cc}
0 & 1 \\
-1 & 0
\end{array}\right], \quad V=\left[\begin{array}{ll}
0 & i \\
i & 0
\end{array}\right], \text { and } W=\left[\begin{array}{cc}
1 & 0 \\
0 & -1
\end{array}\right]
$$

Any two of them anti-commute, $U$ and $V$ are skew-adjoint while $W$ is selfadjoint, and $U^{2}=V^{2}=-I, W^{2}=I$, where $I$ is the $2 \times 2$ identity matrix.

If $n$ is even, $n=2 \mu$, define, for $p=1,2, \ldots, \mu, n$ complex square matrices of size $2^{\mu}$ by the formulas

$$
\begin{aligned}
& E_{2 p-1}:=\underset{1}{W} \otimes \cdots \otimes \underset{p-1}{W} \otimes \underset{p}{U} \otimes \underset{p+1}{I} \otimes \cdots \otimes \underset{\mu}{I}, \\
& E_{2 p}:=\underset{1}{W} \otimes \cdots \otimes \underset{p-1}{W} \otimes \underset{p}{V} \otimes \underset{p+1}{I} \otimes \cdots \otimes \underset{\mu}{I} .
\end{aligned}
$$

If $n$ is odd, $n=2 \mu+1$, in addition to $E_{1}, E_{2}, \ldots, E_{2 \mu}$ given by (2), define

$$
E_{2 \mu+1}:=i \underset{1}{W} \otimes \cdots \otimes \underset{\mu}{W} .
$$

Then $[\mathrm{B}$ the formulas (2) and (3) indeed represent the $n$ generators of an irreducible matrix representation of the complex Clifford algebra $C l\left(\mathbf{R}^{n}\right)$ on $\mathbf{C}^{2^{\mu}}$. The matrices $E_{1}, E_{2}, \ldots, E_{n}$ introduced above have the following useful, easy to check property: All the product matrices $E_{j} E_{k}, j<k$, have 0 as the first element in the first column, except for the $\mu$ matrices $E_{2 p-1} E_{2 p}, p=1,2, \ldots, \mu$, whose first column is precisely $[i, 0, \ldots, 0]^{t}$. Consequently, under the splitting $\mathbf{C}^{2^{\mu}}=\mathbf{C} \times \mathbf{C}^{2^{\mu}-1}$,

$$
\begin{aligned}
& E_{2 p-1} E_{2 p}(\mathbf{C} \times 0)=i \mathbf{C} \times 0, \quad p=1,2, \ldots, \mu, \\
& E_{j} E_{k}(\mathbf{C} \times 0) \subset 0 \times \mathbf{C}^{2^{\mu}-1}, \quad j<k,(j, k) \neq(2 p-1,2 p) .
\end{aligned}
$$

The Euclidean Dirac operator is then the differential operator

$$
\not D: C^{\infty}\left(\Omega, \mathbf{C}^{2^{\mu}}\right) \longrightarrow C^{\infty}\left(\Omega, \mathbf{C}^{2^{\mu}}\right), \quad \Omega \subseteq \mathbf{R}^{n} \text { open, }
$$

defined, for spinors $f \in C^{\infty}\left(\Omega, \mathbf{C}^{2^{\mu}}\right)=C^{\infty}(\Omega, \mathbf{C}) \otimes \mathbf{C}^{2^{\mu}}$ in column form $f=$ $\left[f_{1}, f_{2}, \ldots, f_{2^{\mu}}\right]^{t}, f_{\alpha} \in C^{\infty}(\Omega, \mathbf{C})$, by

$$
\not D f=\sum_{j=1}^{n} E_{j} \nabla_{j} f
$$


where $\nabla_{j}=\frac{\partial}{\partial x_{j}} \otimes I d$ represents ordinary component-wise differentiation with respect to $x_{j}$. It is well known $[\mathrm{LM}]$ that $\not D$ with domain $C_{0}^{\infty}\left(\mathbf{R}^{n}, \mathbf{C}^{2^{\mu}}\right)$ is an essentially selfadjoint first-order elliptic differential operator in $L^{2}\left(\mathbf{R}^{n}, \mathbf{C}^{2^{\mu}}\right)$.

For any magnetic potential $a=\sum_{j=1}^{n} a_{j} d x_{j}$ with real-valued components $a_{j}(x)$ of class $C^{1}$, the operator formally defined by

$$
\not D_{a}:=\sum_{j=1}^{n} E_{j}\left(\nabla_{j}+i a_{j} I d\right)
$$

is a particular instance of a Dirac operator on $\mathbf{R}^{n}$ coupled to a vector potential in the sense of $[\mathrm{LM}]$, that is, a Dirac operator on the trivial tensor bundle $\mathbf{C}^{2^{\mu}} \otimes \mathbf{C}=$ $\mathbf{C}^{2^{\mu}}$ over $\mathbf{R}^{n}$, where $\mathbf{C}$ is equipped with the metric connection $d_{a}=d+i a$. As such, it is again an essentially selfadjoint first-order elliptic differential operator in $L^{2}\left(\mathbf{R}^{n}, \mathbf{C}^{2^{\mu}}\right)$.

For the square of $\not D_{a}$, the following Bochner-Weitzenböck formula LM] holds true on $C^{2}\left(\mathbf{R}^{n}, \mathbf{C}^{2^{\mu}}\right)$,

$$
\not D_{a}^{2}=\left(\nabla \otimes d_{a}\right)^{*}\left(\nabla \otimes d_{a}\right)+\mathcal{R}_{a},
$$

where the connection Laplacian $\left(\nabla \otimes d_{a}\right)^{*}\left(\nabla \otimes d_{a}\right)$ is easily seen to equal $H_{a} \otimes I d$, and where $\mathcal{R}_{a}$ is the Hermitian curvature bundle morphism acting on $\mathbf{C}^{2^{\mu}}$ according to the formula

$$
\mathcal{R}_{a}=\sum_{j<k} E_{j} E_{k} R_{j k}^{a}, \quad R_{j k}^{a}=\left[\nabla_{j}+i a_{j} I d, \nabla_{k}+i a_{k} I d\right]=i B_{j k} I d .
$$

\section{The Proof of the Theorem}

Proof. It is known [I, $\mathrm{KS}$ ] that $H_{a}$ has a discrete spectrum if and only if there is a function $\lambda \in C\left(\mathbf{R}^{n}, \mathbf{R}\right), \lim _{|x| \longrightarrow \infty} \lambda(x)=+\infty$, such that for any $\phi \in C_{0}^{\infty}\left(\mathbf{R}^{n}, \mathbf{C}\right)$,

$$
\left(H_{a} \phi, \phi\right) \geq(\lambda \phi, \phi)
$$

where $(\cdot, \cdot)$ denotes the standard inner product in $L^{2}\left(\mathbf{R}^{n}, \mathbf{C}\right)$.

Assume first that hypothesis (10) of the Theorem is satisfied for $j_{p}=2 p-1$, $k_{p}=2 p, \epsilon_{p}=1, p=1,2, \ldots, \mu$. Applying the Bochner-Weitzenböck identity (5) to the spinor $[\phi, 0,0, \ldots, 0]^{t} \in C_{0}^{\infty}\left(\mathbf{R}^{n}, \mathbf{C}^{2^{\mu}}\right)$ yields, via equations (5) and (4),

$$
\left(H_{a} \phi, \phi\right)=\left\|\not D_{a}[\phi, 0,0, \ldots, 0]^{t}\right\|^{2}+\left(\left(\sum_{p=1}^{\mu} B_{2 p-1,2 p}\right) \phi, \phi\right),
$$

and so the content of equation (17) is fulfilled by letting $\lambda:=\sum_{p=1}^{\mu} B_{2 p-1,2 p}$.

The general case now follows by making the substitutions $E_{j_{p}} \longrightarrow(-1)^{\epsilon_{p}} E_{2 p-1}$, $E_{k_{p}} \longrightarrow E_{2 p}$ (and eventually $\left.E_{l} \longrightarrow E_{2 \mu+1}, l \neq j_{p}, k_{p}\right), p=1,2, \ldots, \mu$, in the representation (2), (3) of the Clifford algebra $C l\left(\mathbf{R}^{n}\right)$ on $\mathbf{C}^{2^{\mu}}$. 


\section{ACKNOWLEDGMENT}

This work was completed while the author visited the Simion Stoilow Mathematical Institute of the Romanian Academy (IMAR) on a Bitdefender Professorship. The author would like to thank IMAR for its hospitality and support.

\section{REFERENCES}

[AHS] J. Avron, I. Herbst, B. Simon, Schrödinger Operators with Magnetic Fields, I. General Interactions, Duke Math. J. 45, 847-883 (1978). MR.518109 (80k:35054)

[B] H. Baum, Spin-Strukturen und Dirac-Operatoren über Pseudoriemannschen Mannigfaltigkeiten, Teubner Verlagsgesellschaft, Leipzig, 1981. MR701244 (84m:58160)

[D] A. Dufresnoy, Un Example de Champ Magnétique dans $\mathbf{R}^{\nu}$, Duke Math. J. 50, 729-734 (1983). MR714827 (84k:78005)

[HM] B. Helffer, A. Mohamed, Caractérisation du Spectre Essentiel de l'Opérateur de Schrödinger avec un Champ Magnétique, Ann. Inst. Fourier 38, 95-112 (1988). MR949012 (90d:35215)

[I] A. Iwatsuka, Magnetic Schrödinger Operators with Compact Resolvent, J. Math. Kyoto Univ. 26, 357-374 (1986). MR.857223 (87j:35287)

[KS] V. Kondratiev, M. Shubin, Discreteness of Spectrum for the Magnetic Schrödinger Operators, Commun. Partial Diff. Eqns. 27, 477-525 (2002). MR1900553 (2003f:35058)

[LM] B. Lawson, M-L. Michelsohn, Spin Geometry, Princeton Univ. Press, Princeton, NJ, 1989. MR.1031992 (91g:53001)

Department of Mathematics, University of North Texas, Denton, Texas 76203

E-mail address: anghel@unt.edu 